\title{
Shock wave therapy for Achilles tendinopathy
}

\author{
Michelle Wilson · Jason Stacy
}

Published online: 26 November 2010

(C) Humana Press 2010

\begin{abstract}
Achilles tendinopathy has been reported as the most common overuse injury in sports medicine clinics (Clement et al. Am J Sports Med 12: 179-184, 1984). Standard treatment regimens include activity modification, heel lifts, arch supports, stretching exercises, nonsteroidal anti-inflammatories, and eccentric loading. There is a lack of consensus regarding treatment. Even so, most athletes will respond to this regimen. However, conservative management will prove to be inadequate for a subset of patients. When conservative therapy fails, other noninvasive treatment measures may be considered. Extracorporeal shock wave therapy (ESWT) has been used in soft-tissue disorders including lateral epicondylitis, plantar fasciitis, and calcific tendonitis of the shoulder. Conclusive evidence recommending ESWT as a treatment for Achilles tendinopathy is lacking. This article explores the role of shock wave therapy in the management of recalcitrant Achilles tendinopathy in patients attempting to avoid an invasive intervention.
\end{abstract}

Keywords Achilles tendonitis - Tendinosis ·

Tendinopathy · Heel pain - Conservative management .

Eccentric loading $\cdot$ Shock wave therapy $\cdot$ Surgical management

\section{Introduction}

Achilles tendon injuries are often frustrating causes of posterior heel pain. These injuries typically occur in those

M. Wilson $(\bowtie) \cdot J$. Stacy

University of South Carolina, Columbia, SC 29211, USA

e-mail: schnooka4@yahoo.com participating in distance running and a variety of sports including soccer, football, tennis, volleyball, basketball, badminton, and handball [14, 21]. Reported estimates of the prevalence of Achilles tendinopathy, however, are $11 \%$ in runners, $9 \%$ in dancers, $5 \%$ in gymnasts, $2 \%$ in tennis players, and $<1 \%$ in football players $[3,6,9,38]$. In the various published reports to date, the mean age of patients with peritendinitis and tendinosis ranged from 24 to 30 years $[3,6,38]$. Despite extensive research, the precise cause and natural history of these injuries remains unclear [27].

Risk factors include biomechanical abnormalities of the lower extremity such as hyperpronation and leg length discrepancy as well as systemic conditions such as obesity, hypertension, diabetes, and endocrinopathies. Extrinsic factors include increase in interval training, excessive hill training, a sudden increase in training intensity, change of surface (soft to hard), excessive mileage, and inappropriate or worn-out footwear [12]. Advanced age, male gender, steroid use, and fluoroquinolones are also associated with this condition [12, 22-24]. From an anatomic perspective, the decreased vascularity of the Achilles tendon may predispose the tendon to damage or rupture.

Damage to the Achilles tendon usually results from chronic overuse. It is the result of accumulative impact loading and repetitive microtrauma to the tendon [12]. Injury begins with inflammatory changes around the tendon (peritendinitis) while the tendon itself remains normal. In the majority of patients, the Achilles tendon does not have a tendon sheath but rather a paratenon. The paratenon is the site of inflammation associated with peritendinitis. Peritendinitis may progresses to tendinosis characterized either by degenerative and inflammatory changes within the tendon or by degenerative changes within the tendon and associated inflammation of the peritendinous tissue, 
respectively [4]. Interestingly, inflammatory changes are present, however, inflammatory mediators are absent making the term Achilles tendonitis a misnomer. Tendinopathy is more of a generic term used to encompass intrinsic and/or extrinsic damage to the tendon. From an anatomic standpoint, tendinopathy describes fraying of the tendon due to a failed healing response of the extracellular matrix $[2,25]$.

\section{Anatomy}

The Achilles tendon is the longest and one of the strongest tendons in the body. It is the confluence of the gastrocnemius, soleus, and plantaris muscles and inserts onto the posterior calcaneus. The Achilles tendon has no tendon sheath (two layers of connective tissue surrounding the tendon) but rather a paratenon (a richly vascularized single layer of cells) [31]. However, a small subset of patients will have a synovial sheath instead of a paratenon [4]. The blood supply is largely derived from blood vessels within the paratenon. The tendon has a relatively avascular area $2-6 \mathrm{~cm}$ proximal to its insertion on the calcaneus. This area of avascularity is the most common site of tendon damage known as noninsertional tendinopathy. When tendinopathy occurs at the junction of bone and tendon, it is referred to as insertional tendinopathy. The retrocalcaneal bursa resides between the tendon and the upper portion of the calcaneus.

\section{Diagnosis}

Differential diagnosis of patients with posterior heel pain includes retrocalcaneal bursitis and superficial tendo Achilles bursitis or "pump bumps" [11, 29]. Achilles tendinopathy will often present with soft-tissue swelling, local tenderness, and crepitus [31]. In early stages, focal swelling and tenderness are limited to a small area (see Fig. 1). Crepitus is the result of an exudation around the tendon and is more commonly found in the acute stage. It can be accentuated by active dorsiflexion and plantar flexion of the foot. Thompson's test is negative. Radiographs rarely show calcification of the soft tissues surrounding the tendon or the tendon itself [29]. The lateral radiograph may show a prominent superior tuberosity of the os calcis. Ultrasounds of athletes with chronic Achilles tendinosis have found that this modality accurately demonstrates both tendonitis and tendinosis of the tendon $[19,20]$.

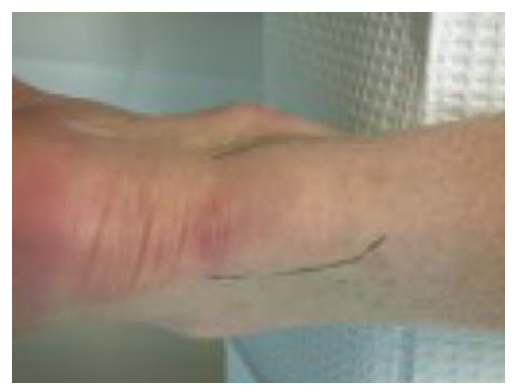

Fig. 1 Achilles tendinopathy

\section{Treatment}

Often medical attention will not be initiated until athletes have attempted to "run through" the pain but have had to stop or severely modify sports activities. Initial treatment of Achilles peritendinitis and tendinosis always is conservative and focuses on control of pain and inflammation, correction of functional malalignment, and rehabilitation of the gastrocnemius-soleus muscle-tendon complex [23]. In athletes who present within 1-2 weeks of the onset of symptoms, a short course (7-10 days) of oral, nonsteroidal anti-inflammatory medications and 2 weeks of rest usually will allow them to return to running, symptom free [31]. In addition, they should be counseled about the extrinsic factors (e.g., errors in training) that may have caused their problem and about prophylactic measures (proper shoes, stretching) that can prevent Achilles tendonitis [23]. Table 1 outlines a common conservative treatment regimen. Immobilization of the ankle for a period of 7-10 days may be indicated in individuals with severe acute symptoms [29]. Steroid injections, however, should be limited to the area of the retrocalcaneal bursa and should be employed only in patients with recalcitrant retrocalcaneal bursitis $[24,25]$. There is a growing amount of evidence that steroid injections in and around the Achilles tendon may increase the risk of tendon rupture $[7,8,26,29]$. The most recent Cochrane review reported insufficient evidence from randomized controlled trials to determine which method of treatment is most appropriate for acute or chronic Achilles tendinopathy. This lack of evidence has led to the exploration of other noninvasive treatment possibilities including ESWT.

A discussion of operative treatment of Achilles tendinopathy is beyond the scope of this article. However, operative treatment should be considered when a comprehensive nonoperative treatment program of several months' duration has failed and the patient is not willing to alter or abandon the precipitating sports activity [39]. Surgical treatment is required in about $25 \%$ of athletes with 
Table 1 Nonoperative treatments

\begin{tabular}{l}
\hline Rest \\
Shoe ware modification \\
Nonsteroidal anti-inflammatory medications \\
Physical therapy \\
Eccentric training \\
Massage \\
Ultrasound \\
Ice \\
Iontophoresis \\
Heel lift orthosis \\
Custom orthosis \\
Immobilization
\end{tabular}

Achilles tendon overuse injuries [41]. Researchers report excellent or good results in up to $85 \%$ of cases, but success rates are not always so high in routine nonspecialized clinical practice $[30,40]$. It is difficult to compare the results of studies as most workers do not report their assessment procedure [40]. Also, no prospective randomized studies comparing operative and conservative treatment of Achilles tendinopathy have been published and most of the knowledge on treatment efficacy comes from clinical experience and descriptive studies [31].

\section{Extracorporeal shock wave therapy}

Extracorporeal shock wave therapy (ESWT) was originally used for the treatment of renal stones. In the 1990s, ESWT became popular in Germany for certain soft-tissue disorders, including calcifying tendonitis of the rotator cuff, humeral epicondylitis, and plantar fasciitis [10]. It is now applied worldwide for the treatment of musculoskeletal complaints. In the USA, the Federal Drug Administration approved the use of ESWT in the treatment of chronic proximal plantar fasciitis in 2000. Shockwaves are pressure disturbances that propagate rapidly though a medium [10]. Attenuation of the wave is dependent on the medium used. In air, a wave dissipates quickly. However, water is an excellent medium. Most treatment protocols use a water medium and a coupling gel before penetrating tissue. Generation of shock waves is electromagnetic, electrohydraulic, piezoelectric, or pneumatic. The first three methods focus the wave to a very specific area, whereas, focusing is not involved using the pneumatic method [10]. In animal models, ESWT stimulates soft-tissue healing primarily by inhibiting afferent pain-receptor function and by enhancing angiogenesis. Basic science studies show that ESWT may increase blood flow to the treated site and induce an inflammatory-mediated healing process.
Treatment protocols utilize either low- or high-energy waves. Low-energy shock wave therapy does not require local anesthesia but does require three to four applications. In contrast, high-energy shock wave therapy can be applied in one visit but requires local anesthesia. Controversy revolves around whether the use of anesthesia compromises clinical outcomes [35]. In theory, the infiltration of local anesthesia may interfere with the focusing of the shock wave to the site of maximal discomfort [35]. Also local anesthesia may alter the inflammatory response and angiogenesis ESWT invokes. Regional anesthesia may be of use as it can provide comfort for the patient without compromising the results of SWT. The type of anesthesia that is most beneficial has yet to be determined.

The application of ESWT varies widely in the existing trials making comparison of results difficult. Conflicting results may be related to differing treatment protocols. The trials conducted for lateral epicondylitis, plantar fasciitis, and calcific tendonitis of the shoulder generally use a protocol, in which 1,500-2,000 shocks of low energy are applied to the site of maximal discomfort. Local anesthesia is not used. Applications are done at weekly intervals for three to four applications, and patients are followed for at least 3-4 months after last application [37]. This protocol has been used in ESWT for Achilles tendinopathy trials. In contrast and perhaps complicating, trials utilizing high energy one time application of ESWT with anesthesia have also been conducted $[1,17,18]$. Chen et al. found that in rat Achilles tendons low-energy shock wave therapy restored biomechanical and biomechanical characteristics of healing tendon while high-energy SWT resulted in inhibitory effects on repair.

Uncontrolled pilot studies suggested that SWT can be an effective treatment for patients with chronic noninsertional Achilles tendinopathy [28, 33]. More recent randomized controlled trials have substantiated these positive results [5, $32,36]$. In the largest of these studies Rompe et al. enrolled 75 patients in a randomized triple-arm trial to compare eccentric loading, repetitive low-energy SWT, and a waitand-see policy. SWT was administered without local anesthesia in weekly intervals with a primary follow-up of at least 12 weeks. At 4 months from baseline, 60 vs. 52 vs. $24 \%$ reported symptoms "completely recovered" or "much improved" [36]. For all outcome measures, eccentric loading and SWT did not differ significantly but showed significantly better results than the wait-and-see policy [36].

Furia $[15,16]$ studied SWT for both chronic insertional and noninsertional Achilles tendinopathy in two separate trials. In these case controlled studies, single dose, highenergy SWT with anesthesia was applied. The insertional Achilles tendinopathy study had 68 participants. A group of 35 patients received one dose of high-energy SWT. The 
other group of 33 patients received nonoperative treatment. Visual analog scale and Roles and Maudsley score were used for evaluation. Patients were followed up at 1, 3, and 12 months after treatment. The VAS scores at 1,3 , and 12 months for nonoperative therapy and ESWT were 8.2 and 4.2, 7.2 and 2.9, and 7.0 and 2.8, respectively [15]. Twelve months after treatment $83 \%$ of patients receiving SWT had improvement of their symptoms according to the Roles and Maudsley score. A subgroup of patients receiving SWT had regional anesthesia rather than local. The percentage of patients with successful Roles and Maudsley scores did not differ in these patients. This study concluded that ESWT is an effective treatment for chronic insertional Achilles tendinopathy [15]. It further stated that local anesthesia may decrease the effectiveness of the procedure. The second of these case controlled trials focused on noninsertional chronic Achilles tendinopathy. In this trial, 33 patients received a single dose of highenergy shock wave therapy with regional anesthesia. Thirty-four patients were treated with eccentric training. VAS and Roles and Maudsley score were again used to evaluate outcomes. Patients were followed up at 1, 3, and 12 months after treatment. VAS at 1,3 , and 12 months for nonoperative therapy and SWT were 8.4 and 4.4, 6.5 and 2.9 , and 5.6 and 2.2, respectively [16]. The percentage of excellent or good Roles and Maudsley scores at 12 months were statistically greater in the SWT group than in the control group. The authors concluded that ESWT is an effective treatment for chronic noninsertional Achilles tendinopathy [16].

Two double-blinded, randomized controlled trials have been completed. Costa et al. [13] reported no statistically significant treatment effects in 49 patients with Achilles tendinopathy treated with low-energy SWT. Patients with insertional and noninsertional forms of Achilles tendinopathy were included in the trial. Each patient was treated in 3 monthly intervals with primary follow-up 4 weeks after SWT. Baseline visual analog scale for pain during walking was 55 for both SWT and control groups. The score after intervention was 34 points in the SWT group and 50 in the control group [13]. Although their findings were not statistically significant, the authors acknowledge that "the confidence intervals include the potential for a clinically relevant treatment effect." This raises the possibility of type II error, that is, the sample size was too small to detect a meaningful treatment effect [13].

Rasmussen et al. [34] compared low-energy ESWT versus sham therapy. Participants, 48 in all, were randomized to receive active or sham ESWT over 4 weeks. All patients received nonoperative therapy. Each treatment group consisted of 24 patients. American Orthopaedic Foot and Ankle Society (AOFAS) score and pain were used for evaluation. Patients were followed up at 4,8 , and
12 weeks. AOFAS increased more over time in the intervention group than in the control, from 74 to 81 in the placebo group and from 70 to 88 in the intervention group [34]. Better results were seen in the intervention group at 8 and 12 weeks follow-up. Pain was reduced in both groups but no statistically significant difference was noted. Interestingly, women showed the best outcome, an unexpected finding. The authors concluded that ESWT appears to be a supplement for the treatment of chronic Achilles tendinopathy [34].

\section{Conclusion}

Clearly, more clinical trials are needed to evaluate the efficacy of SWT for Achilles tendinopathy. In the Cochrane review referenced earlier, only nine clinical trials for a total of 697 patients were of sufficient quality to be considered. This was a review of all treatments of Achilles tendinopathy not only ESWT. However, it does point out the need for higher numbers of participants. Studies comparing high energy, single-treatment protocols with low energy, multiple-treatment protocols, and studies comparing various dosing intervals and energy flux densities are also needed to determine optimal treatment parameters. A standardized method to evaluate results may also be helpful. However, it does appear that ESWT is a reasonable adjunct to therapy when conservative treatment fails.

\section{References}

1. Albert JD, Meadeb J, Guggenbuhl P, Marin F, Benkalfate F, Thomazeu H, Chales G. High-energy extracorporeal shock-wave therapy for calcifying tendinitis of the rotator cuff. J Bone Joint Surg. 2007;89(3):335-41.

2. Alfredson H, Pietila T, Honsson P, Lorentzon R. Heavy-load eccentric calf muscle raining for the treatment of chronic Achilles tendinosis. Am J Sports Med. 1998;26:360-6.

3. Amiel D, Frank CB, Harwood FL, et al. Tendons and ligaments: a morphological and biochemical comparison. J Orthop Res. 1984; $1: 257-65$

4. Arner O, Lindholm A, Orell SR. Histologic changes in subcutaneous rupture of the Achilles tendon. Acta Chir Scand. 1958;116: 484-90.

5. Astore F, Sansone V, De Marchi F. Extracorporeal shock wave therapy for tendinopathy of Achilles. Read at the 23rd Isokinetic congress on rehabilitation of sports muscle and tendon injuries, Turin, Italy; 2004.

6. Astrom M, Westlin N. No effect of piroxicam on chronic Achilles tendinopathy: a randomized study of 70 patients. Acta Orthop Scand. 1992;63:631-4.

7. Balasubramaniam $P$, Prathap K. The effect of injection of hydrocortisone into rabbit calcaneal tendons. J Bone Joint Surg Br. 1972;54:729-34.

8. Barnett T, Hammond NL. Anterior tibial tendon rupture. Contemp Orthop. 1991;23:365-7. 
9. Caine D, Cochrane B, Caine C, Zemper E. An epidemiologic investigation of injuries affecting young competitive female gymnasts. Am J Sports Med. 1989;17:811-20.

10. Chung B, Wiley JP. Extracorporeal shockwave therapy: a review. Sports Med. 2002;32:851-65.

11. Clancy WG Jr, Neidhart D, Brand RL. Achilles tendonitis in runners: a report of five cases. Am J Sports Med. 1976;4:46-57.

12. Clement DB, Taunton JE, Smart GW. Achilles tendonitis and peritendinitis: etiology and treatment. Am J Sports Med. 1984;12: 179-84.

13. Costa ML, Shepstone L, Donell ST, Thomas TL. Shock wave therapy for chronic Achilles tendon pain: a randomized placebocontrolled trial. Clin Orthop Relat Res. 2005;440:199-204.

14. Fox JM, Blazina ME, Jobe FW, et al. Degeneration and rupture of the Achilles tendon. Clin Orthop. 1975;107:221-4.

15. Furia JP. Extracorporeal shockwave therapy in the treatment of chronic insertional Achilles tendinopathy. Orthopade. 2005;34: 571-8.

16. Furia JP. Extracorporeal shockwave therapy in the treatment of chronic noninsertional Achilles tendinopathy. Am J Sports Med. 2006;34(5):502-8.

17. Furia JP. High-energy extracorporeal shock wave therapy as a treatment for insertional Achilles tendinopathy. Am J Sports Med. 2006;34(5):733-40.

18. Furia JP. High-energy extracorporeal shock wave therapy as a treatment for chronic noninsertional Achilles tendinopathy. Am J Sports Med. 2008;36(3):502-8.

19. Gibbon WW, Cooper JR, Radcliffe GS. Sonographic incidence of tendon microtears in athletes with chronic Achilles tendinosis. $\mathrm{Br}$ J Sports Med. 1999;33:129-30.

20. Gibbon WW, Cooper JR, Radcliffe GS. Distribution of sonographically detected tendon abnormalities in patients with a clinical diagnosis of chronic Achilles tendinosis. J Clin Ultrasound. 2000;28:61-6.

21. Griffiths JC. Tendon injuries around the ankle. J Bone Joint Surg Br. 1965;47:686-9.

22. James SL, Bates BT, Ostering LR. Injuries to runner. Am J Sports Med. 1978;6:40-5.

23. Jarvinen TA, Kannus P, Paavola M, Jarvinen TL, Jozsa L, Jarvinen M. Achilles tendon injuries. Curr Opin Rheumatol. 2001;13(2):150-5.

24. Jones DC. Achilles tendon problems in runners. Instr Course Lect. 1998;47:419-27.

25. Khan KM, Cook JL, Kannus P, Maffulli N, Bonar SF. Time to abandon the "tendinitis" myth. BMJ. 2002;324:626-7.

26. Kleinman M, Gross AE. Achilles tendon rupture following steroid injection. Report of three cases. J Bone Joint Surg Am. 1983;65:1345-7.
27. Kvist M. Achilles injuries in athletes. Sports Med. 1994;18: 173-201.

28. Lakshmanan P, O'Doherty DP. Chronic Achilles tendinopathy: treatment with extracorporeal shock wave. Foot Ankle Surg. 2004; $10: 125-30$

29. Leach RE, James S, Wasilewski S. Achilles tendinitis. Am J Sports Med. 1981;9:93-8.

30. Maffulli N, Binfield P, Moore D, et al. Surgical decompression of chronic central core lesions of the Achilles tendon. Am J Sports Med. 1999;27:747-52.

31. Maffulli N, Sharma P, Luscombe KL. Achilles tendinopathy: etiology and management. J R Soc Med. 2004;97:472-6.

32. Peers K. Extracorporeal shock wave therapy in chronic Achilles and patellar tendinopathy. Leuven: Leuven University Press; 2003.

33. Perlick L, Schiffmann R, Kraft CN, Wallny T, Diedrich O. Extracorporeal shock wave treatment of the Achilles tendonitis: experimental and preliminary clinical results. Z Orthop Ihre Grenzgeb. 2002;140(3):275-80.

34. Rasmussen S, Christensen M, Mathiesen I, Simonson O. Shockwave therapy for chronic Achilles tendinopathy: a doubleblind, randomized clinical trial of efficacy. Acta Orthop. 2008; 79(2):249-56.

35. Rompe JD, Schoellner C, Nafe B, Hofmann A, Gerdesmeyer L. Repetitive low-energy shock wave application without local anesthesia is more efficient than repetitive low-energy shock wave application with local anesthesia in the treatment of chronic plantar fasciitis. J Orthop Res. 2005;23:931-41.

36. Rompe JD, Nafe B, Furia J, Maffulli N. Eccentric loading, shock wave treatment or a wait-and-see policy for tendinopathy of the main body of the tendo Achilles: a randomized controlled trial. Am J Sports Med. 2007;35:374-83.

37. Rompe JD, Furia J, Maffulli N. Eccentric loading compared with shock wave therapy for chronic insertional Achilles tendinopathy. J Bone Joint Surg Am. 2008;90(1):52-61.

38. Saltin B, Henricksson J, Nygaard E, Anderson P. Fiber types and metabolic action potentials of skeletal muscles in sedentary man and endurance runners. Ann N Y Acad Sci. 1977;310:3-29.

39. Schepsis AA, Leach RE. Surgical management of Achilles tendonitis. Am J Sports Med. 1987;15:308-15.

40. Tallon D, Coleman D, Khan KM, Maffuli N. Outcome of surgery for chronic Achilles tendinopathy: a critical review. Am J Sports Med. 2001;29:315-20.

41. Woodley BL, Newsham-West RJ, Baxter GD. Chronic tendinopathy: effectiveness of eccentric exercise. Br J Sports Med. 2007;41(4):188-98. 